\title{
Interdependencia de los mercados de valores en el mundo*
}

\author{
Interdependence of the Stock Markets around the World \\ Elena Moreno Garcia, ${ }^{* *}$ Daniel Vázquez Cotera, ${ }^{* * *}$ \\ Sergio Hernández Mejía**** y Luis Alberto Larios Ojeda*****
}

\section{RESUMEN}

Se presenta el análisis de los rendimientos de los mercados de valores de mayor capitalización en el mundo por zona horaria para el período 2009-2015, incluyendo a la Bolsa Mexicana de Valores. Se utilizó la prueba de causalidad de Granger para identificar al mercado líder y encontrar un orden con base en la exogeneidad de cada mercado. Se empleó el modelo de vectores autorregresivos con sus respectivas pruebas de simulación: el análisis impulso-respuesta y la descomposición de la varianza del error de pronóstico para comprobar la rapidez de adaptación, la respuesta de los mercados ante movimientos externos y la proporción de dichos cambios que se atribuye a cada mercado. Se halló evidencia de fuerte interdependencia entre las bolsas, rapida respuesta a movimientos externos y liderazgo del mercado estadounidense.

Palabras clave: bolsas de valores, capitalización, mercados financieros internacionales, interdependencia, impactos externos.

Clasificación JEL: G14, G15.

\begin{abstract}
This paper shows the analysis of the most capitalized stock markets returns of each time zone for the period 2009 - 2015. It was included in the analysis the Mexican stock market. Granger's causality test was employed to recognize a market that acts as leader and to find an order based on the exogeneity of each market. The Vector Autoregression Model was used, with its simulation tests: the impulse-response analysis and decomposition of variance of forecast error to check the speed of adaptation, market response to external shocks and the proportion of such shocks attributed to each market. There is evidence of the strong interdependence between the world's major stocks markets, the position of the EU market as a leader and the markets fast response to external shocks.

Keywords: stock markets, capitalization, international financial markets, interdependence, external shocks.

JEL classification: G14, G15.

* Fecha de recepción: 22/08/2013.. Fecha de aprobación: 08/07/2015.

** Directora de la División de Negocios y Economía de la Universidad Cristóbal Colón. Correo electrónico: moreno.garciae12@gmail.com.

*** Asesor en asuntos económicos de la rectoría de la Universidad Cristóbal Colón: Correo electrónico: dcotera@ucc.mx.

**** Jefe de la Licenciatura en Economía de la Universidad Cristóbal Colón. Correo electrónico: sergiohm@ucc.mx.

***** Ejecutivo senior business banking en HSBC. Correo electrónico: luislarios@hotmail.com.
\end{abstract}




\section{INTRODUCCIÓN}

Existe un gran número de estudios e investigaciones que vinculan el desarrollo de los mercados financieros con el crecimiento económico y detallan la importancia y los beneficios que traen consigo el desarrollo y crecimiento de estos mercados para la economía de un país. Lanteri (2011); Brugger y Ortiz (2012), y Walker (1998) afirman que el desarrollo del mercado financiero de un país es condición necesaria para alcanzar mejores tasas de crecimiento económico.

Considerando que hay un vínculo importante entre el desarrollo del mercado financiero de un país y sus tasas de crecimiento económico, en la literatura ha habido numerosos estudios enfocados a medir el desarrollo de los mercados financieros en general y de los mercados de valores en particular. Beck, Demirguc-Kunt y Levine (2000) han identificado que el indicador más empleado para medir el tamaño de los mercados financieros es la razón de capitalización del mercado como porcentaje del producto interno bruto (PIB); como indicador de actividad se ocupa la proporción del valor total de valores negociados como porcentaje del PIB y como indicador de eficiencia, el índice de rotación del mercado de valores. Dentro de los indicadores sugeridos para medir el desarrollo del mercado de valores, la capitalización del mercado como porcentaje del PIB es el que más se ocupa. Algunos ejemplos de estudios que usan este indicador para realizar comparaciones entre países son: Bayraktar (2014); Atje y Jovanovic (1993); Demirguc-Kunt y Levine (1996); Levine y Zervos (1998); Garcia y Liu (1999); Arestis, Demetriadis y Luintel (2001); Naceur, Ghazouani y Omran (2007); Billmeier y Massa (2009); Beck y Demirgüç-Kunt (2009); Beck et al. (2010); Narayan, Mishra y Narayan (2011); Wang, Medianu y Whally (2011); Cihak, Demirguc-Kunt, Feyen y Levine (2012); Barajas, Chami y Yousefi (2013a), y Barajas, Beck, Dabla-Norris y Yousefi (2013).

La Federación Internacional de Bolsas de Valores mide la importancia de los mercados accionarios en las economías a través de un indicador que compara la relación existente entre el nivel de capitalización bursátil y el PIB. En 2011, la capitalización del NYSE representaba 78.3\% de la economía estadounidense y si se sumara la capitalización del NASDAQ, el porcentaje que representarían los mercados accionarios americanos sería muy superior a su producto interno burto. En México la significación del mercado de valores sobre la economía es de $34.5 \%$, así mismo, Zavaleta y Urbina (2011), concluyen que en el largo plazo el desarrollo del mercado accionario explica parte del crecimiento de la economía real en México. 
Otro indicador de la evolución de los mercados accionarios es el valor de las operaciones bursátiles, definido como el número total de acciones comerciadas multiplicado por sus respectivos precios (Federación Internacional de Bolsas de Valores, 2010). En el período 2000-2011 el valor de los títulos negociados en los mercados de valores mundiales creció $64.5 \%$. Por zona horaria, América permanece como la región dominante alcanzando en 2010 un importe operado de USD 50933441.7 millones. ${ }^{1}$ Cabe señalar que la proporción del valor de las operaciones bursátiles de la zona horaria Asia-Pacifico con respecto al total de las tres zonas se ha más que duplicado en este período.

El número total de operaciones en todas las bolsas ha crecido en $596 \%$ durante el período 2000-2011, siendo la región de Asia-Pacífico la que realiza el mayor número de transacciones, seguida por América con 6297184900 y 5348 477900 transacciones en 2011, respectivamente.

No sólo el crecimiento de los mercados de valores ha sido significativo, también la integración de los mercados en las últimas décadas ha sido extraordinario, hecho que es producto de diversos factores, como la globalización financiera, la desregulación de los mercados y la eficiencia en la transmisión de la información entre otros. Los mercados de valores han evolucionado abriendo sus puertas a nuevas formas de negociación, a inversionistas y empresas provenientes de otros mercados, ampliando así sus alcances y objetivos, conduciendo a un aumento súbito de las relaciones entre los mismos (Rigobon, Ehrmann y Fratzcher, 2005).

Existe amplia evidencia de que los mercados se han vuelto más interconectados en las últimas décadas si se considera el volumen de comercialización transfronteriza y los flujos de capital (véase Forbes y Chinn, 2004). Angkinand, Barth y Kim (2009) encontraron evidencia de varios grados de interdependencia entre los rendimientos de los mercados de valores de varios países a través del tiempo. Aunque no hubo completa uniformidad en los resultados de todos y cada uno de los países, no hay duda de que ha habido un cambio en el grado de interdependencia entre los diferentes países y los Estados Unidos a través del tiempo.

Dado el creciente nivel de operaciones bursátiles a nivel global y su influencia en la economía de los países, el presente trabajo se plantea los siguientes objetivos: 1) Analizar la relación de los mercados de valores más capitalizados en el mundo por zona horaria (Brasil, Estados Unidos, Reino Unido, Shangai y

${ }^{1}$ El fuerte incremento de la zona de América en 2007-2008 se debe parcialmente a un cambio metodológico de las bolsas de Estados Unidos. 
Japón), con inclusion del mexicano, durante el periodo 2009-2015. 2) Identificar a un mercado que actúe como líder de los demás. 3) Analizar la proporción de los movimientos en un mercado que puedan ser explicados por los movimientos en otro.

El análisis de la información se realiza considerando la teoría de la eficiencia de los mercados que está asociada con la idea de una "caminata aleatoria", término que se ha empleado para caracterizar series de precios en las que todos los cambios de precios posteriores representan desviaciones aleatorias de los precios anteriores (Malkiel, 2003). A continuación, en la sección I se enuncian las hipótesis de la investigación y se describe la metodología empleada en el trabajo. La II presenta el análisis de los resultados y, finalmente, se plantean las conclusiones.

\section{HIPÓTESIS Y METODOLOGÍA}

H1: Los mercados de México, Reino Unido, Estados Unidos, Brasil, Shangai y Japón mantienen una correlación positiva especialmente para aquellos que se encuentran más cercanos entre sí.

H2: La bolsa de valores de Estados Unidos actúa como mercado líder, afectando significativamente a todos los demás mercados.

H3: Existe una rápida velocidad de adaptación a impactos externos por parte de las bolsas de valores gracias a una ágil asimilación y procesamiento de la información de acuerdo a la teoría de la eficiencia de los mercados.

\section{Descripción metodológica}

En el presente análisis, las variables de estudio son los rendimientos de las principales bolsas de valores del mundo de acuerdo a la zona horaria en la que se encuentran. Se eligieron para el análisis las más capitalizadas por zona horaria, de acuerdo a la información que en 2011 presentó la Federación Internacional de Bolsas de Valores.

Se siguen de cerca los trabajos de Eun y Shim (1989); Villanueva (2008), y Peña (1991), al incorporar técnicas econométricas como la prueba de causalidad de Granger, con el objeto de identificar un mercado que actúe como líder y para determinar un orden con base en la exogeneidad de cada mercado. También 
se empleó el modelo de vectores autorregresivos (VAR), con sus respectivas pruebas de simulación: el análisis impulso-respuesta y la descomposición de la varianza del error de pronóstico, para comprobar la rapidez de adaptación, la respuesta de los mercados ante impactos externos y la proporción de dicho impacto que se atribuye a cada mercado.

Se aplicó la metodología ocupada por Climent, Meneu, y Pardo (1999) mediante el análisis de regresiones multivariantes por mínimos cuadrados ordinarios con el objeto de evaluar la significación estadística y sentido de las relaciones entre las bolsas de valores, considerando la zona horaria en la que se encuentran.

Como variable indirecta se utilizaron los rendimientos de los índices bursátiles de Brasil, Estados Unidos, Reino Unido, Japón, Shangai y México: IBOVESPA, Dow Jones Industrial Average (DJIA), Financial Times Stock Exchange (FTSE 100), Nikkei 225, sSE Composite Index e Índice de Precios y Cotizaciones (IPC), respectivamente. Los datos empleados son los valores al cierre de cada índice, los cuales son transformados en rendimientos porcentuales diarios. Para ello se ha tomado el rendimiento o la rentabilidad, como la diferencia de logaritmos de los precios de cierre de cada mercado. Esto es, la rentabilidad de un índice $j$ en un instante de tiempo $t$ :

$$
R_{t}^{j}=\ln \left(P_{t}^{j}\right)-\ln \left(P_{t-1}^{j}\right)
$$

siendo $P_{t}^{j}$ el precio de cierre del índice $j$ en el período $t$. Esta transformación también contribuye a evitar problemas potenciales vinculados a la no estacionariedad de las series originales de los índices bursátiles (Villanueva, 2008).

Se ocupan datos diarios debido a que, de acuerdo con Eun y Shim (1989), un mes o incluso una semana puede ser un período de tiempo lo suficientemente largo como para atenuar las interacciones que sólo duren unos pocos días. Ocupar datos diarios permitirá capturar también estas interacciones entre las bolsas, las cuales concuerdan con la teoría de la eficiencia de los mercados. Para los datos faltantes debido a días festivos o no laborales en un mercado, mientras los otros están abiertos, se sigue la metodología de Liu, Pan y Shieh (1998) ajustando las series de tiempo al eliminar los datos sobrantes con el fin de asegurar que cada bolsa de valores tenga una observación en cada fecha del período considerado.

Con la intención de evitar el efecto confuso que puede generar la devaluación de la moneda local en una región sobre los resultados del estudio, todos 
los rendimientos de los índices bursátiles están expresados en moneda local, tal como lo sugieren Yang, Hsiao, Li, y Wang (2005). Anteriormente, la literatura usaba normalmente datos convertidos a dólares estadounidenses con el propósito de reflejar el punto de vista de un inversionista y de hacer los rendimientos comparables entre sí. Sin embargo, en las publicaciones más recientes, algunos investigadores ocupan datos en dólares estadounidenses mientras que otros usan datos expresados en moneda local. Koch y Koch (1991); Chen, Firth, y Rui (2002), y Bessler y Yang (2003) incluyeron en sus modelos datos medidos tanto en dólares estadounidenses como en moneda local y encontraron cambios muy pequeños en los resultados.

Considerando lo anterior, se pretende hacer uso de la matriz de correlaciones y de la regresión lineal múltiple para encontrar la correlación existente entre pares de mercados y entre un mercado determinado con respecto a los demás. Se utiliza el método de mínimos cuadrados ordinarios (MCO) para estimar una regresión lineal múltiple y se propone como variable dependiente los rendimientos diarios del Índice de Precios y Cotizaciones, y como variables explicativas las variaciones de los índices DJIA, Ibovespa, FTSE, Nikkei y SSE Composite Index.

Posteriormente, se realizaron pruebas de raíz unitaria utilizando la prueba de Dickey-Fuller aumentada (ADF) para comprobar la estacionariedad de las series y mediante los criterios de Akaike, Schwarz y Hannan-Quinn se esperaba encontrar el número de rezagos óptimos a incluir en el modelo. Peña (1991), Villanueva (2008) y Assaf (2003) sugieren que cinco rezagos son necesarios, ya que de esta manera se asegura que toda la dinámica de los datos sea capturada y usada en el análisis. También, para evitar problemas derivados de la estimación de un modelo infraparametrizado, se ha utilizado un número fijo de retardos para todas las variables explicativas del modelo (Gredenhoff y Karlson, 1999).

Así mismo, a través del prueba de causalidad de Granger, se encontraron las relaciones causales entre las bolsas con el objetivo de comprobar la existencia de un mercado que funja como "mercado líder" y determinar el orden con base en la exogeneidad que se usa en el modelo var. Una ventaja importante de la prueba de causalidad de Granger es que no es afectado por el orden de las variables debido a que cada prueba se realiza por pares de variables (Friedman y Shachmurove, 1997), por lo que los resultados de esta prueba sirven para determinar la exogeneidad relativa de las variables y de esta manera establecer su ordenación, tal y como lo sugieren Tsangyao y Chien-Chung (2001).

A pesar de la importancia teórica que se le da al orden en que se introducen las variables al sistema, Soydemir (2000) menciona en su trabajo que varios 
estudios que ocupan datos de alta frecuencia, como datos diarios, para mercados bursátiles desarrollados, no encuentran evidencia de que los resultados sean sensibles al orden de las variables, pues datos con una periodicidad mayor tienden a suavizar las series. Además, Mills y Mills (1991) utilizan en su investigación dos órdenes diferentes, uno basado en los horarios de negociación y otro basado en la metodología usada por Eun y Shim (1989), sin encontrar diferencias significativas en los resultados.

Finalmente, se estimó un modelo VAR, ya que es adecuado cuando la teoría económica no establece una relación clara entre las variables objeto de estudio. Es apropiado también porque revela el grado de interdependencia entre los mercados, además de la importancia relativa de ellos, explicando variaciones o impactos inesperados en otros. Así como también, es capaz de simular la respuesta de un mercado ante innovaciones en otro para inferir la rapidez en la transmisión de dichos impactos, probando al mismo tiempo los efectos de contagio e interdependencia. Sin embargo, como ya se mencionó, dada la complejidad en la interpretación de los parámetros de los modelos VAR, se recurre al análisis de las simulaciones propuestas por Sims (1980). La primera de ellas es la descomposición de la varianza del error de pronóstico. Si bien la prueba de causalidad de Granger indica si otros mercados causan en sentido Granger a un mercado dado y viceversa, es posible que otros mercados puedan influenciar a ese mercado a través de otras ecuaciones en el sistema (Friedman y Shachmurove, 1997). Por esta razón es que se utilizó también el análisis de descomposición de varianza para determinar la influencia y proporción de los movimientos en un mercado de valores que puede ser explicada por innovaciones en otros mercados.

De igual manera, a partir del modelo var, se utilizaron las funciones de impulso-respuesta, así como sus bandas de confianza para otorgar evidencia de la velocidad con la que los movimientos de las bolsas son transmitidos al resto de los mercados. Runkle (1987) menciona que el reportar las funciones impulsorespuesta sin los intervalos de confianza, equivale a reportar los coeficientes de una regresión sin el estadístico $t$ de Student, por lo que en este estudio se trazan las bandas de confianza para cada función impulso-respuesta. Para la interpretación de los resultados, Assaf (2003) señala que si la respuesta de un mercado determinado ante un impacto en otro se disipa rápidamente, entonces la transmisión de la información entre estos mercados es relativamente eficiente.

El análisis se realiza con un total de 1299 observaciones, del período comprendido entre el 5 de enero de 2009 y el 30 de abril de 2015, con el fin de analizar una época, lo más actual posible, libre de crisis económicas. Como se ha 
mencionado, la zona horaria en la que se encuentra cada bolsa de valores es un determinante importante en la especificación de los modelos y en la interpretación de los resultados, por lo tanto, los horarios de negociación que se consideran para cada mercado se presentan en el cuadro 1.

Cuadro 1. Horarios de negociación

\begin{tabular}{lcc}
\hline \multicolumn{1}{c}{ Mercado de valores } & Horario local de negociación & Diferencia horaria \\
\hline New York Stock Exchange & $9: 30-16: 00$ & GMT - 5 \\
London Stock Exchange & $8: 30-16: 30$ & GMT \\
Bolsa de Tokio & $9: 00-11: 00$ y 12:30 -15:00 & GMT + 9 \\
Bolsa de Valores de Sao Paulo & $10: 00-5: 30$ & GMT - 3 \\
Bolsa Mexicana de Valores & $8: 30-15: 00$ & GMT - 6 \\
Bolsa de Valores de Shangai & $9: 30-16: 00$ & GMT + 8 \\
\hline
\end{tabular}

Fuente: Elaboración propia con datos de las respectivas bolsas de valores.

Las series de tiempo de los índices bursátiles diarios de cada mercado fueron obtenidas de la agencia de información estadounidense Bloomberg. De la muestra, se extrajeron los días festivos o no laborales y fueron transformadas en rendimientos porcentuales diarios mediante diferencia de logaritmos.

\section{ANÁLISIS DE RESULTADOS}

Una vez que se consiguieron las series de tiempo de la muestra elegida para el período seleccionado, se procedió al análisis econométrico de los datos ya transformados a diferencias de logaritmos. ${ }^{2}$ El cuadro 2 muestra los estadísticos principales de las series de tiempo a analizar.

Considerando 1299 observaciones de cada serie, se puede notar que la media de los rendimientos de los índices es muy cercana a cero en todos los casos. El IBOVESPA es el que presenta la mayor volatilidad, mientras que el IPC es el menos volátil. La literatura especializada indica que generalmente los rendimientos de los activos financieros presentan características de leptocurtosis acentuadas y los datos del presente estudio no son la excepción.

\footnotetext{
${ }^{2}$ Para el análisis de las series de tiempo se utilizaron los paquetes econométricos RATS, E-views y GRETL.
} 
Cuadro 2. Estadísticos principales

(05/01/2009-30/04/2015)

\begin{tabular}{lcccccr}
\hline & IBOVESPA & DJIA & FTSE & IPC & \multicolumn{1}{c}{ Nikkei } & SSE \\
\hline Media & 0.023085 & 0.016257 & 0.019096 & 0.049538 & -0.006082 & 0.042582 \\
Mediana & 0.043487 & 0.045875 & 0.028631 & 0.112262 & 0.025694 & 0.050994 \\
Máximo & 5.746151 & 6.611575 & 5.032250 & 5.862618 & 5.522320 & 5.935971 \\
Mínimo & -8.430565 & -5.706112 & -5.480507 & -6.062028 & -11.15343 & -6.214640 \\
Desv. Est. & 1.577958 & 1.229912 & 1.256058 & 1.196553 & 1.458869 & 1.374093 \\
Sesgo & -0.096473 & -0.176694 & -0.140061 & -0.367014 & -0.578795 & -0.233203 \\
Curtosis & 5.003922 & 6.376725 & 4.923822 & 6.790340 & 7.977441 & 5.304720 \\
Jarque-Bera & 140.8394 & 400.5686 & 131.3397 & 517.9653 & 907.4940 & 299.2716 \\
Probabilidad & 0.000000 & 0.000000 & 0.000000 & 0.000000 & 0.000000 & 0.000000 \\
Observaciones & 1299 & 1299 & 1299 & 1299 & 1299 & 1299 \\
\hline
\end{tabular}

La matriz de correlaciones que se presenta en el cuadro 3, evidencia la importancia de la zona horaria ya que los pares de mercados que exhiben una mayor correlación son aquellos cuyos horarios de negociación son más cercanos, es decir, se detecta que a mayor cercanía geográfica existe un mayor nivel de correlación lineal.

La mayor correlación se encuentra entre el IPC y el DJIA (0.712545) y de igual manera se encuentran altas correlaciones entre los pares DJIA e IBOVESPA, IPC e IBOVESPA, y DJIA y FTSE, las cuales son mayores a 0.6. Por otro lado, los índices Nikkei y SSE son los que muestran los niveles de correlación más bajos con respecto a los demás.

Cuadro 3. Matriz de coeficientes de correlación

\begin{tabular}{ccccccl}
\hline DJIA & FTSE & Nikkei & IBOVESPA & IPC & SSE & \\
\hline \multirow{2}{*}{1.0000} & 0.700960 & 0.136870 & 0.637442 & 0.712545 & 0.104234 & DJIA \\
& 1.0000 & 0.248299 & 0.540570 & 0.579153 & 0.179210 & FTSE \\
& & 1.0000 & 0.106081 & 0.166911 & 0.273572 & Nikkei \\
& & & 1.0000 & 0.637442 & 0.178847 & IBOVESPA \\
& & & & 1.0000 & 0.184625 & IPC \\
& & & & & 1.0000 & SSE \\
\hline
\end{tabular}

Mientras el análisis de correlación permite vincular los mercados por pares y proporciona una idea intuitiva y estática de la dependencia de los mismos, con el análisis de regresión múltiple es posible estudiar la relación de un mercado en particular con respecto al resto de los mercados conjuntamente. Cen- 
trándose en las relaciones que guarda el mercado bursátil mexicano con el resto de las bolsas incluidas en la muestra, se presentan en el cuadro 4 los resultados de la estimación por MCO.

Cuadro 4. Regresión lineal múltiple por MCO

(variable dependiente: IPC, $n=834$ )

\begin{tabular}{lccccc}
\hline & Coeficiente & Desv. Típica & Estadístico $t$ & \multicolumn{2}{c}{ Valor de $p$} \\
\hline Constante & 0.012199 & 0.020320 & 0.600316 & 0.5484 & \\
DJ & 0.480343 & 0.029276 & 16.40751 & $<0.00001$ & $* * *$ \\
SSE & 0.049841 & 0.015607 & 3.193561 & 0.0014 & $* * *$ \\
IBOVESPA & 0.202422 & 0.017239 & 11.74193 & $<0.00001$ & $* * *$ \\
FTSE & 0.081517 & 0.026677 & 3.057949 & 0.00044 & $* * *$ \\
Nikkei & 0.027611 & 0.014588 & 1.892784 & 0.06415 & $*$ \\
\hline Media de la vble. dep. & 0.032177 & D.T. de la vble. dep. & 1.119896 \\
Suma de cuad. residuos & 691.5231 & D.T. de la regresión & 0.731314 \\
$R^{2}$ & 0.575208 & R-cuadrado corregido & 0.573565 \\
F(5,1,299) & 350.1685 & Valor de $p$ (de $F$ ) & 0.000000 \\
Log-verosimilitud & -1433.722 & Criterio de Akaike & 2.216662 \\
Criterio de Schwarz & 2.240538 & Crit. de Hannan-Quinn & 2.225621 \\
& & \multicolumn{2}{l}{ Durbin-Watson } & 1.865309 \\
\hline
\end{tabular}

Los parámetros estimados son todos positivos y estadísticamente significativos en $1 \%$, con excepción del Nikkei, el cual tiene una significación de $10 \%$. La probabilidad del estadístico $F$ demuestra que todas las variables independientes son estadísticamente significativas de manera conjunta, por lo que es posible afirmar que las variables empleadas explican en gran medida el comportamiento del IPC. Así mismo, es interesante observar que los valores de los parámetros son más grandes a medida que existe un mayor sincronismo horario, con lo que se confirma nuevamente la primera hipótesis. El modelo presenta un valor de $R^{2}$ ajustado aceptable (0.57), y por el valor del estadístico Durbin-Watson, cercano a 2 , se prueba que el modelo estimado no viola el supuesto de no autocorrelación.

Con esta primera aproximación, se comprueba que la relación lineal que guarda la Bolsa Mexicana de Valores con el resto de las bolsas es positiva y que es mayor en la medida en que aumenta la coincidencia de los horarios de negociación. En este sentido, la bolsa estadounidense mostró el coeficiente más grande, probando ser la que más influye sobre el mercado mexicano. 
Para el análisis por el método VAR es necesario comprobar la estacionariedad de las series empleadas en el modelo, por lo que se recurre a las pruebas de raíz unitaria mediante la prueba $\mathrm{ADF}$, cuyos resultados aparecen en el cuadro 5.

Cuadro 5. Prueba de Dickey-Fuller aumentada (ADF)

\begin{tabular}{|c|c|c|c|}
\hline \multicolumn{4}{|c|}{ IBOVESPA } \\
\hline \multirow[t]{3}{*}{ Prueba estadística ADF } & \multirow[t]{3}{*}{-38.08961} & $1 \%$ Valor crítico* & -3.4351 \\
\hline & & $5 \%$ Valor crítico & -2.8635 \\
\hline & & $10 \%$ Valor crítico & -2.5678 \\
\hline \multicolumn{4}{|c|}{ DJIA } \\
\hline \multirow[t]{3}{*}{ Prueba estadística ADF } & -24.16555 & $1 \%$ Valor crítico* & -3.4409 \\
\hline & & $5 \%$ Valor crítico & -2.8654 \\
\hline & & $10 \%$ Valor crítico & -2.5688 \\
\hline \multicolumn{4}{|c|}{ FTSE } \\
\hline \multirow[t]{3}{*}{ Prueba estadística ADF } & -36.56297 & $1 \%$ Valor crítico* & -3.4351 \\
\hline & & $5 \%$ Valor crítico & -2.8635 \\
\hline & & $10 \%$ Valor crítico & -2.5678 \\
\hline \multicolumn{4}{|c|}{ IPC } \\
\hline \multirow[t]{3}{*}{ Prueba estadística ADF } & -33.94387 & $1 \%$ Valor crítico* & -3.4351 \\
\hline & & $5 \%$ Valor crítico & -2.8635 \\
\hline & & $10 \%$ Valor crítico & -2.5678 \\
\hline \multicolumn{4}{|c|}{ Nikkei } \\
\hline \multirow[t]{3}{*}{ Prueba estadística ADF } & -36.74927 & $1 \%$ Valor crítico* & -3.4351 \\
\hline & & $5 \%$ Valor crítico & -2.8635 \\
\hline & & $10 \%$ Valor crítico & -2.5678 \\
\hline \multicolumn{4}{|c|}{ SSE } \\
\hline \multirow[t]{3}{*}{ Prueba estadística ADF } & -36.73932 & $1 \%$ Valor crítico* & -3.4351 \\
\hline & & $5 \%$ Valor crítico & -2.8635 \\
\hline & & $10 \%$ Valor crítico & -2.5678 \\
\hline
\end{tabular}

Los resultados de esta prueba apuntan a la estacionariedad de todas las series temporales que se emplearán en el modelo. El valor absoluto del estadístico de MacKinnon es mucho menor que el valor absoluto del estadístico $t$ en todos los casos, por lo que se rechaza la presencia de raíz unitaria. Dado que las series temporales de los rendimientos de los índices bursátiles se han obtenido a 
través de la primera diferencia de logaritmos, se dice que son series integradas de orden uno y es posible hacer uso de ellas para estimar el modelo var.

Una decisión vital para el análisis es la selección del número óptimo de rezagos a incluir en el modelo. Se utilizaron los criterios de Akaike, Schwarz y de Hannan-Quinn como guías para la decisión. De acuerdo a los dos últimos se debería incluir un rezago, mientras que bajo el criterio de Akaike el número óptimo de retardos es dos. Se realizó el prueba hasta por 36 rezagos, sin cambio alguno en los resultados aunque en el cuadro 6 se presentan sólo diez retardos.

Cuadro 6. Número óptimo de rezagos

\begin{tabular}{ccccc}
\hline Retardos & Log. Veros. & AIC & BIC & HQC \\
\hline 1 & -10705 & 17.0186 & $17.1896^{*}$ & $17.0829^{*}$ \\
2 & -10666 & $17.0142^{*}$ & 17.3317 & 17.1335 \\
3 & -10645 & 17.0382 & 17.5022 & 17.2127 \\
4 & -10616 & 17.04937 & 17.6599 & 17.2788 \\
5 & -10595 & 17.0731 & 17.8303 & 17.4388 \\
6 & -10576 & 17.0993 & 18.0030 & 17.5030 \\
7 & -10545 & 17.1084 & 18.1586 & 17.5578 \\
8 & -10509 & 17.1078 & 18.3045 & 17.6461 \\
9 & -10494 & 17.1414 & 18.4847 & 17.7406 \\
10 & -10483 & 17.1807 & 18.6706 & 17.8136 \\
\hline
\end{tabular}

Nota: Los asteriscos indican los mejores valores (los mínimos) de cada criterio de información. $\mathrm{AIC}=$ criterio de Akaike, BIC = criterio bayesiano de Schwarz y HQC = criterio de HannanQuinn.

Dentro de la literatura revisada, la gran mayoría de las investigaciones relacionadas con este tema utilizan el criterio de Akaike para elegir el número de rezagos a incluir, pues mencionan que la mayoría de los contrastes son extensiones de dicho criterio y son altamente sensibles a no normalidad, y dada la forma leptocúrtica que generalmente se presenta en las distribuciones de las series financieras, el criterio de Akaike parece ser la mejor opción.

Se prosigue con la prueba de causalidad de Granger que se presenta en el cuadro 7, con el objetivo de encontrar las relaciones entre los índices e identificar un mercado líder, así como para determinar el orden de exogeneidad de las variables, con base en un análisis objetivo de las series. 
Cuadro 7. Prueba de causalidad de Granger con dos rezagos

\begin{tabular}{|c|c|c|c|}
\hline Hipótesis Nula: & Obs & Estadístico $F$ & Probabilidad \\
\hline DJIA no causa en el sentido Granger a IBOVESPA & 1297 & 3.2676 & 0.0384 \\
\hline IBOVESPA no causa en el sentido Granger a DJIA & & 0.8918 & 0.4101 \\
\hline FTSE no causa en el sentido Granger a IBOVESPA & 1297 & 1.1948 & 0.3031 \\
\hline IBOVESPA no causa en el sentido Granger a FTSE & & 8.6964 & 0.0002 \\
\hline IPC no causa en el sentido Granger a IBOVESPA & 1297 & 0.3027 & 0.7389 \\
\hline IBOVESPA no causa en el sentido Granger a IPC & & 0.7046 & 0.4945 \\
\hline SSE no causa en el sentido de Granger a IBOVESPA & 1297 & 0.3191 & 0.7268 \\
\hline IBOVESPA no causa en el sentido de Granger a SSE & & 20.068 & 0.0000 \\
\hline Nikkei no causa en el sentido Granger a IBOVESPA & 1297 & 0.1024 & 0.9026 \\
\hline IBOVESPA no causa en el sentido Granger a Nikkei & & 76.333 & 0.0000 \\
\hline FTSE no causa en el sentido Granger a DJIA & 1297 & 5.1837 & 0.0057 \\
\hline DJIA no causa en el sentido Granger a FTSE & & 13.8435 & 0.0000 \\
\hline IPC no causa en el sentido Granger a DJIA & 1297 & 3.9918 & 0.0187 \\
\hline DJIA no causa en el sentido Granger a IPC & & 6.2629 & 0.0020 \\
\hline Nikkei no causa en el sentido Granger a DJIA & 1297 & 0.68191 & 0.5058 \\
\hline DJIA no causa en el sentido Granger a Nikkei & & 153.932 & 0.0000 \\
\hline SSE no causa en el sentido de Granger a DJIA & 1297 & 0.41487 & 0.6605 \\
\hline DJIA no causa en el sentido de Granger a SSE & & 17.2984 & 0.0000 \\
\hline IPC no causa en el sentido Granger a FTSE & 1297 & 12.7897 & 0.3426 \\
\hline FTSE no causa en el sentido Granger a IPC & & 1.07209 & 0.0000 \\
\hline Nikkei no causa en el sentido Granger a FTSE & 1297 & 0.4562 & 0.6337 \\
\hline FTSE no causa en el sentido Granger a Nikkei & & 99.717 & 0.0000 \\
\hline SSE no causa en el sentido Granger a FTSE & 1297 & 0.40477 & 0.6672 \\
\hline FTSE no causa en el sentido Granger a SSE & & 11.5630 & 0.0000 \\
\hline SSE no causa en el sentido Granger a Nikkei & 1297 & 0.41942 & 0.6575 \\
\hline Nikkei no causa en el sentido Granger a SSE & & 2.28473 & 0.1022 \\
\hline SSE no causa en el sentido Granger a IPC & 1297 & 0.71132 & 0.4912 \\
\hline IPC no causa en el sentido Granger a SSE & & 14.5651 & 0.0000 \\
\hline Nikkei no causa en el sentido Granger a IPC & 1297 & 0.2841 & 0.7527 \\
\hline IPC no causa en el sentido Granger a Nikkei & & 73.519 & 0.0000 \\
\hline
\end{tabular}


Por los resultados del cuadro 7, es evidente el liderazgo del mercado estadounidense, dado que el DJIA causa en sentido Granger a todos los demás índices, mientras que ninguno es capaz por sí solo de afectarlo, lo que concuerda con la teoría revisada y con la segunda hipótesis planteada. Destaca el hecho de que la bolsa de Brasil, logra causar en sentido Granger a las de México, Londres, Japón y Shangai, por lo que se coloca como la segunda bolsa más exógena de acuerdo a esta prueba. Le sigue el IPC, al causar en sentido Granger al FTSE, Nikkei y al SSE, mientras que el mercado inglés sólo causa en sentido Granger al mercado nipón. Finalmente, los índices Nikkei y sSE resultaron como los mercados más endógenos, ya que no afectan a ningún mercado pero sí son causados por los demás.

El orden con base en la exogeneidad queda de la siguiente manera: DJIA, IBOVESPA, IPC, FTSE, Nikkei y SSE. Es posible que la zona horaria esté jugando un papel importante en las relaciones de causalidad antes descritas, pues se debe notar que el orden derivado de la prueba de Granger es prácticamente de acuerdo a la región donde se ubican las bolsas de valores, lo que también concuerda con el orden seleccionado por otros autores como Meneu y Climent (2001), quienes utilizan el criterio de zona horaria en vez de exogeneidad.

Seleccionado ya el orden de las variables, se puede realizar el análisis mediante el método VAR. El modelo planteado estima un sistema dinámico de ecuaciones simultáneas, libre de restricciones a priori sobre la relación de las variables, por lo que evita errores de especificación en el modelo.

A pesar de que el cirterio de Akaike indicó que incluir dos rezagos en el modelo es suficiente, la diferencia del valor del AIC para los rezagos dos y cinco es tan pequeña que resulta difícil discriminar con claridad entre las alternativas. Por ello, y con un criterio conservador, se eligió un orden de retardo suficientemente largo como para abarcar una semana de actividad, lo cual parece suficiente para el propósito del trabajo.

Los resultados del modelo var se presentan en el cuadro A1 del anexo. Para su interpretación se utilizan la descomposición de la varianza del error de predicción y las funciones de impulso-respuesta, además se toma en consideración el no sincronismo de los horarios de negociación de los mercados, por lo que se examina cuidadosamente la estructura de las diferencias horarias y se incorporan sus implicaciones al análisis de los resultados. El cuadro 8 muestra la descomposición de varianzas para un horizonte temporal de 1, 5, 10, 15 y 20 días, periodo suficiente para analizar toda la dinámica de adaptación en las bolsas de valores. 
Cuadro 8. Descomposición de la varianza del error de predicción

\begin{tabular}{|c|c|c|c|c|c|c|c|c|}
\hline $\begin{array}{l}\text { Mercado } \\
\text { Explicado }\end{array}$ & Horizonte & D.E. & DJIA & IBOVESPA & IPC & FTSE & Nikkei & SSE \\
\hline \multirow[t]{5}{*}{ DJIA } & 1 & 1.059573 & 100.0000 & 0.000000 & 0.000000 & 0.000000 & 0.000000 & 0.000000 \\
\hline & 5 & 1.083854 & 97.69851 & 0.185321 & 0.792863 & 0.702012 & 0.406179 & 0.215114 \\
\hline & 10 & 1.092176 & 96.54923 & 0.342991 & 0.787843 & 0.927611 & 0.404942 & 0.987386 \\
\hline & 15 & 1.092257 & 96.54357 & 0.343772 & 0.790139 & 0.927786 & 0.405470 & 0.989263 \\
\hline & 20 & 1.092258 & 96.54350 & 0.343791 & 0.790146 & 0.927787 & 0.405471 & 0.989307 \\
\hline \multirow[t]{5}{*}{ IBOVESPA } & 1 & 1.549297 & 39.66231 & 60.33769 & 0.000000 & 0.000000 & 0.000000 & 0.000000 \\
\hline & 5 & 1.570020 & 39.20344 & 58.94601 & 0.885057 & 0.634343 & 0.295170 & 0.035987 \\
\hline & 10 & 1.580199 & 38.87178 & 58.43815 & 0.933598 & 0.664446 & 0.297087 & 0.794935 \\
\hline & 15 & 1.580287 & 38.87253 & 58.43320 & 0.936038 & 0.664507 & 0.297161 & 0.796564 \\
\hline & 20 & 1.580288 & 38.87252 & 58.43316 & 0.936045 & 0.664510 & 0.297162 & 0.796607 \\
\hline \multirow[t]{5}{*}{ IPC } & 1 & 1.110145 & 51.30749 & 5.923047 & 42.76947 & 0.000000 & 0.000000 & 0.000000 \\
\hline & 5 & 1.123169 & 50.39881 & 5.968132 & 42.75391 & 0.161394 & 0.508261 & 0.209494 \\
\hline & 10 & 1.128574 & 50.20689 & 6.058683 & 42.37120 & 0.194069 & 0.514154 & 0.655005 \\
\hline & 15 & 1.128626 & 50.20487 & 6.059417 & 42.36804 & 0.194436 & 0.514230 & 0.659002 \\
\hline & 20 & 1.128627 & 50.20486 & 6.059423 & 42.36801 & 0.194437 & 0.514231 & 0.659043 \\
\hline \multirow[t]{5}{*}{ FTSE } & 1 & 1.091766 & 52.48176 & 1.708485 & 0.230757 & 45.57899 & 0.000000 & 0.000000 \\
\hline & 5 & 1.117322 & 51.10738 & 1.950728 & 0.765983 & 45.23924 & 0.304323 & 0.632338 \\
\hline & 10 & 1.123443 & 51.18255 & 1.979566 & 0.790337 & 44.78329 & 0.377789 & 0.886470 \\
\hline & 15 & 1.123517 & 51.18225 & 1.980638 & 0.791145 & 44.77829 & 0.378119 & 0.889557 \\
\hline & 20 & 1.123518 & 51.18225 & 1.980648 & 0.791158 & 44.77824 & 0.378126 & 0.889588 \\
\hline \multirow[t]{5}{*}{ Nikkei } & 1 & 1.314284 & 3.681863 & 0.126325 & 0.618897 & 2.084156 & 93.48876 & 0.000000 \\
\hline & 5 & 1.482631 & 20.15372 & 0.734837 & 0.665847 & 2.333362 & 75.46475 & 0.647477 \\
\hline & 10 & 1.491652 & 20.07181 & 0.779854 & 0.725251 & 2.418802 & 74.70494 & 1.299341 \\
\hline & 15 & 1.491738 & 20.07306 & 0.780278 & 0.726321 & 2.420610 & 74.69761 & 1.302127 \\
\hline & 20 & 1.491739 & 20.07306 & 0.780286 & 0.726331 & 2.420645 & 74.69752 & 1.302156 \\
\hline \multirow[t]{5}{*}{ SSE } & 1 & 1.346942 & 1.309190 & 2.232107 & 1.098819 & 0.494960 & 2.989491 & 91.87543 \\
\hline & 5 & 1.384428 & 4.055056 & 2.937775 & 1.295998 & 0.840242 & 3.464671 & 87.40626 \\
\hline & 10 & 1.386980 & 4.199728 & 2.956922 & 1.380202 & 0.850512 & 3.462359 & 87.15028 \\
\hline & 15 & 1.387010 & 4.200618 & 2.957691 & 1.380331 & 0.850562 & 3.462404 & 87.14839 \\
\hline & 20 & 1.387011 & 4.200627 & 2.957696 & 1.380334 & 0.850568 & 3.462404 & 87.14837 \\
\hline
\end{tabular}


Nuevamente se muestra el DJIA como el mercado más exógeno debido a que más de $96 \%$ de sus variaciones son explicadas por sí mismo, mientras que ninguna bolsa por sí sola llega a explicar $1.0 \%$ del mercado estadounidense y conjuntamente llegan a explicar poco más del 3\%.

Además, el DJIA logra explicar buena parte de las variaciones de los demás mercados, $39 \%$ del mercado brasileño, $50 \%$ del mexicano, $51 \%$ del inglés, $20 \%$ del japonés y $4 \%$ del mercado de Shangai. Se confirma una vez más la hipótesis sobre el liderazgo y la exogeneidad de la bolsa estadounidense, ya que influye pero no es influida por otros mercados.

Cabe señalar, que en el caso del SSE, la varianza explicada por el DJIA es menor en comparación a las registradas en las otras bolsas, hecho que puede deberse a la diferencia en los horarios de apertura y cierre de los mercados. Por la misma razón, la variabilidad mostrada en el mercado bursátil de Shangai es explicada en $91 \%$ por sí mismo en el primer período y disminuye a $87 \%$ en períodos posteriores.

Para el caso de la Bolsa Mexicana de Valores, el segundo mercado más influyente es el de Brasil, el cual explica más de $5 \%$ de su volatilidad, contribuyendo así a que más de $67 \%$ de las variaciones del IPC sean explicadas por mercados externos.

Finalmente, el análisis de las funciones impulso-respuesta ofrece una manera adicional para examinar la respuesta de cada una de las bolsas ante innovaciones en otros mercados. Los resultados gráficos se presentan en la gráfica A1 del anexo, los cuales muestran las respuestas de cada mercado ante innovaciones en el resto de las bolsas.

Cabe mencionar que se realizaron varias pruebas con diferente número de rezagos, tanto para la función impulso-respuesta como para el análisis de la descomposición de varianza, sin encontrar grandes diferencias en los resultados, prueba de que la elección de cinco rezagos es más que suficiente para el estudio, dado que la velocidad con la que se adaptan los mercados de acuerdo a los resultados no sobrepasa un periodo de una semana. En el analisis realizado, la reacción de todos los mercados se disipa en dos o máximo tres jornadas bursátiles, por lo que los resultados de la prueba función impulso-respuesta son consistentes con la noción de los mercados internacionales eficientes.

La primera característica que salta a la vista es que el DJIA no reacciona significativamente a innovaciones ocurridas en otras bolsas de valores, mientras que impactos ocurridos en el mercado estadounidense son percibidos significativamente por todos los mercados el mismo día en que se generan. Destaca el he- 
cho que la respuesta del mercado bursatil de Japón y Shangai ante impactos de Estados Unidos alcancen un mayor nivel en el día dos. Esto se debe a la diferencia horaria, ya que estos mercados asiáticos cierran antes de que el estadounidense abra por lo que reacciona con un día de retraso, lo que coincide con los resultados encontrados por Eun y Shim (1989).

\section{CONCLUSiones}

Los resultados obtenidos coinciden con las hipótesis del trabajo que planteaban que los mercados de México, Reino Unido, Estados Unidos, Brasil, Shangai y Japón mantienen una correlación positiva especialmente para aquellos que se encuentran más cercanos entre sí; que la bolsa de valores de Estados Unidos actúa como mercado líder, afectando significativamente a todos los demás mercados, y que existe una rápida velocidad de adaptación a movimientos externos por parte de las bolsas de valores gracias a una ágil asimilación y procesamiento de la información de acuerdo a la teoría de la eficiencia de los mercados.

También se hizo evidente que a mayor cercanía geográfica entre mercados, existe un mayor nivel de correlación lineal y que la mayor correlación se encuentra entre los de México y Estados Unidos En este sentido, la bolsa estadounidense mostró el coeficiente más grande, probando ser la que más influye sobre el mercado mexicano. Las bolsas de Japón y Shangai, en cambio, mostraron los niveles de correlación más bajos con respecto a los demás índices y en el caso particular del índice de Shangai la varianza explicada por el DJIA es menor en comparación a las registradas en las otras bolsas, hecho que puede deberse a la diferencia en los horarios de apertura y cierre de los mercados.

Se confirma la hipótesis sobre el liderazgo y la exogeneidad del mercado estadounidense, ya que logra explicar buena parte de las variaciones de los demás mercados: 39\% del mercado brasileño, 50\% del mexicano, $51 \%$ del inglés, $20 \%$ del japonés y $4 \%$ del mercado de Shangai. De esta forma, se identifica al DJIA como el índice más exógeno, debido a que más de $96 \%$ de sus variaciones son explicadas por sí mismo, mientras que ninguna bolsa por sí sola llega a explicar $1.0 \%$ del mercado estadounidense y conjuntamente llegan a explicar poco más de 3\%. Es decir, la primera característica que salta a la vista es que el DJIA no reacciona significativamente a innovaciones ocurridas en otras bolsas de valores, mientras que movimientos ocurridos en el mercado estadounidense son percibidos significativamente por todos los mercados el mismo día en que se generan. 
Estos resultados aportan evidencia de que la diversificación internacional de los portafolios de inversión no contribuye a una reducción significativa del riesgo de inversión debido a la alta interdependecia entre las bolsas. El análisis realizado se limitó a ciertos mercados considerados como los principales por ser las bolsas más capitalizadas de cada zona horaria. Se propone el estudio de los canales de transmisión, la segmentación por regiones y la inclusión de otros mercados en el modelo como futuros temas de investigación, así como también el análisis del contraste entre períodos de estabilidad económica y períodos de crisis. Finalmente, se sugiere el empleo de diferentes técnicas econométricas, como el análisis de cointegración, para el estudio e interpretación de los resultados.

\section{Anexo}

Cuadro A1. Estimación del modelo de vectores autorresgresivos

\begin{tabular}{ccccccc}
\hline & DJIA & IBOVESPA & IPC & \multicolumn{1}{l}{ FTSE } & Nikkei & \multicolumn{1}{c}{ SSE } \\
\hline DJIA(-1) & -0.211962 & -0.188216 & -0.113448 & 0.115031 & 0.449510 & 0.133516 \\
& $(0.04893)$ & $(0.07154)$ & $(0.05126)$ & $(0.05041)$ & $(0.06069)$ & $(0.06220)$ \\
& {$[-4.33226]$} & {$[-2.63092]$} & {$[-2.21312]$} & {$[2.28178]$} & {$[7.40691]$} & {$[2.14671]$} \\
\hline DJIA(-2) & 0.113029 & 0.183650 & 0.049145 & 0.113276 & 0.071811 & 0.179593 \\
& $(0.05142)$ & $(0.07519)$ & $(0.05388)$ & $(0.05298)$ & $(0.06378)$ & $(0.06537)$ \\
& {$[2.19811]$} & {$[2.44255]$} & {$[0.91220]$} & {$[2.13795]$} & {$[1.12587]$} & {$[2.74745]$} \\
\hline \multirow{2}{*}{ DJIA(-3) } & 0.028602 & -0.006718 & -0.017612 & 0.035437 & 0.073546 & -0.005684 \\
& $(0.05164)$ & $(0.07550)$ & $(0.05410)$ & $(0.05320)$ & $(0.06405)$ & $(0.06564)$ \\
& {$[0.55393]$} & {$[-0.08898]$} & {$[-0.32555]$} & {$[0.66606]$} & {$[1.14830]$} & {$[-0.08659]$} \\
\hline \multirow{2}{*}{ DJIA(-4) } & 0.008826 & -0.136202 & 0.041753 & 0.033179 & 0.008073 & 0.003552 \\
& $(0.05145)$ & $(0.07523)$ & $(0.05391)$ & $(0.05302)$ & $(0.06382)$ & $(0.06541)$ \\
& {$[0.17153]$} & {$[-1.81037]$} & {$[0.77451]$} & {$[0.62582]$} & {$[0.12649]$} & {$[0.05431]$} \\
\hline \multirow{2}{*}{ DJIA(-5) } & 0.030722 & -0.005084 & -0.001725 & -0.000732 & -0.023163 & 0.028899 \\
& $(0.04992)$ & $(0.07299)$ & $(0.05230)$ & $(0.05144)$ & $(0.06192)$ & $(0.06346)$ \\
& {$[0.61543]$} & {$[-0.06965]$} & {$[-0.03298]$} & {$[-0.01423]$} & {$[-0.37408]$} & {$[0.45539]$} \\
\hline \multirow{2}{*}{ IBOVESPA(-1) } & -0.004742 & -0.042646 & 0.007622 & 0.054832 & 0.079456 & 0.097201 \\
& $(0.02689)$ & $(0.03932)$ & $(0.02817)$ & $(0.02771)$ & $(0.03335)$ & $(0.03418)$ \\
& {$[-0.17637]$} & {$[-1.08467]$} & {$[0.27053]$} & {$[1.97907]$} & {$[2.38230]$} & {$[2.84366]$} \\
& & & & &
\end{tabular}




\begin{tabular}{|c|c|c|c|c|c|c|}
\hline & DJIA & IBOVESPA & IPC & FTSE & Nikkei & SSE \\
\hline \multirow[t]{3}{*}{ IBOVESPA $(-2)$} & -0.033047 & -0.026507 & 0.005018 & 0.017265 & -0.025791 & -0.010606 \\
\hline & $(0.02703)$ & $(0.03952)$ & $(0.02832)$ & $(0.02785)$ & $(0.03352)$ & $(0.03436)$ \\
\hline & {$[-1.22280]$} & {$[-0.67076]$} & {$[0.17721]$} & [0.62000] & {$[-0.76935]$} & {$[-0.30872]$} \\
\hline \multirow[t]{3}{*}{ IBOVESPA(-3) } & -0.019336 & 0.022434 & -0.006209 & -0.000174 & -0.050503 & 0.020471 \\
\hline & $(0.02703)$ & $(0.03952)$ & $(0.02832)$ & $(0.02785)$ & $(0.03352)$ & $(0.03436)$ \\
\hline & {$[-0.71543]$} & [0.56769] & {$[-0.21926]$} & {$[-0.00625]$} & {$[-1.50649]$} & [0.59583] \\
\hline \multirow[t]{3}{*}{ IBOVESPA(-4) } & -0.013129 & -0.007346 & 0.008318 & -0.027957 & -0.042185 & 0.006364 \\
\hline & $(0.02706)$ & $(0.03957)$ & $(0.02835)$ & $(0.02788)$ & $(0.03356)$ & $(0.03440)$ \\
\hline & {$[-0.48518]$} & {$[-0.18567]$} & [0.29339] & {$[-1.00271]$} & {$[-1.25682]$} & [0.18502] \\
\hline \multirow[t]{3}{*}{ IBOVESPA(-5) } & -0.014268 & -0.040801 & -0.022798 & -0.003443 & 0.006220 & 0.002452 \\
\hline & $(0.02684)$ & $(0.03924)$ & $(0.02812)$ & $(0.02765)$ & $(0.03329)$ & $(0.03411)$ \\
\hline & {$[-0.53170]$} & [-1.03982] & {$[-0.81086]$} & {$[-0.12454]$} & [0.18686] & [0.07189] \\
\hline \multirow[t]{3}{*}{$\operatorname{IPC}(-1)$} & 0.076858 & 0.083532 & 0.127562 & 0.079251 & -0.02603 & 0.027439 \\
\hline & $(0.04140)$ & $(0.06053)$ & $(0.04337)$ & $(0.04265)$ & $(0.05135)$ & $(0.05262)$ \\
\hline & [1.85664] & [1.38003] & [2.94112] & [1.85801] & {$[-0.50694]$} & [0.52143] \\
\hline \multirow[t]{3}{*}{$\mathrm{IPC}(-2)$} & 0.008335 & -0.078378 & -0.067808 & 0.014397 & 0.005590 & -0.061149 \\
\hline & $(0.04141)$ & $(0.06055)$ & $(0.04339)$ & $(0.04267)$ & $(0.05136)$ & $(0.05264)$ \\
\hline & [0.20129] & [-1.29447] & {$[-1.56292]$} & [0.33742] & [0.10884] & {$[-1.16165]$} \\
\hline \multirow[t]{3}{*}{$\operatorname{IPC}(-3)$} & -0.036852 & -0.040205 & 0.008049 & -0.04079 & -0.022234 & 0.080647 \\
\hline & $(0.04142)$ & $(0.06056)$ & $(0.04339)$ & $(0.04267)$ & $(0.05137)$ & $(0.05265)$ \\
\hline & {$[-0.88978]$} & {$[-0.66390]$} & [0.18548] & {$[-0.95583]$} & {$[-0.43280]$} & [1.53177] \\
\hline \multirow[t]{3}{*}{ IPC(-4) } & 0.105206 & 0.150365 & 0.052807 & 0.104205 & 0.065920 & 0.060610 \\
\hline & $(0.04115)$ & $(0.06018)$ & $(0.04312)$ & $(0.04241)$ & $(0.05105)$ & $(0.05232)$ \\
\hline & [2.55634] & [2.49875] & [1.22469] & [2.45737] & [1.29132] & [1.15853] \\
\hline \multirow[t]{3}{*}{ IPC(-5) } & 0.005456 & -0.022213 & -0.013875 & -0.042213 & -0.010259 & -0.078293 \\
\hline & $(0.04095)$ & $(0.05988)$ & $(0.04291)$ & $(0.04220)$ & $(0.05080)$ & $(0.05206)$ \\
\hline & [0.13322] & {$[-0.37094]$} & {$[-0.32336]$} & {$[-1.00035]$} & {$[-0.20195]$} & {$[-1.50388]$} \\
\hline \multirow[t]{3}{*}{$\operatorname{FTSE}(-1)$} & 0.092250 & 0.127541 & -0.005779 & -0.183816 & 0.145668 & -0.011353 \\
\hline & $(0.04085)$ & $(0.05974)$ & $(0.04280)$ & $(0.04210)$ & $(0.05068)$ & $(0.05193)$ \\
\hline & [2.25801] & [2.13503] & {$[-0.13501]$} & {$[-4.36662]$} & [2.87453] & {$[-0.21860]$} \\
\hline $\operatorname{FTSE}(-2)$ & -0.04024 & -0.074696 & 0.000701 & -0.121234 & 0.079981 & -0.097741 \\
\hline
\end{tabular}


174 ECONOMÍA: TEORÍA Y PRÁCTICA • Nueva Época, número 43, julio-diciembre 2015

\begin{tabular}{|c|c|c|c|c|c|c|}
\hline & DJIA & IBOVESPA & IPC & FTSE & Nikkei & SSE \\
\hline & $(0.04254)$ & $(0.06220)$ & $(0.04457)$ & $(0.04383)$ & $(0.05277)$ & $(0.05408)$ \\
\hline & {$[-0.94594]$} & {$[-1.20086]$} & [0.01572] & [-2.76583] & [1.51577] & [-1.80743] \\
\hline \multirow[t]{3}{*}{ FTSE $(-3)$} & -0.016182 & -0.015174 & -0.007326 & -0.009692 & 0.033260 & -0.076298 \\
\hline & $(0.04284)$ & $(0.06263)$ & $(0.04488)$ & $(0.04414)$ & $(0.05313)$ & $(0.05445)$ \\
\hline & {$[-0.37777]$} & {$[-0.24226]$} & {$[-0.16324]$} & {$[-0.21958]$} & [0.62597] & {$[-1.40116]$} \\
\hline \multirow[t]{3}{*}{ FTSE $(-4)$} & -0.02667 & 0.034062 & -0.038314 & -0.001223 & 0.115266 & 0.008157 \\
\hline & $(0.04259)$ & $(0.06228)$ & $(0.04462)$ & $(0.04389)$ & $(0.05283)$ & $(0.05414)$ \\
\hline & {$[-0.62619]$} & [0.54695] & {$[-0.85858]$} & {$[-0.02786]$} & [2.18181] & [0.15066] \\
\hline \multirow[t]{3}{*}{ FTSE $(-5)$} & -0.058582 & 0.009806 & 0.026506 & -0.007982 & 0.078740 & 0.012171 \\
\hline & $(0.04098)$ & $(0.05992)$ & $(0.04293)$ & $(0.04222)$ & $(0.05083)$ & $(0.05209)$ \\
\hline & {$[-1.42961]$} & [0.16365] & [0.61739] & {$[-0.18904]$} & [1.54916] & {$[0.23365]$} \\
\hline \multirow[t]{3}{*}{ Nikkei(-1) } & 0.023886 & -0.037845 & 0.004086 & 0.009883 & -0.133132 & -0.068981 \\
\hline & $(0.02370)$ & $(0.03465)$ & $(0.02483)$ & $(0.02442)$ & $(0.02939)$ & $(0.03012)$ \\
\hline & [1.00806] & {$[-1.09229]$} & [0.16460] & [0.40477] & {$[-4.52960]$} & {$[-2.29006]$} \\
\hline \multirow[t]{3}{*}{ Nikkei(-2) } & 0.017430 & 0.030670 & 0.011312 & -0.00334 & -0.038379 & -0.048564 \\
\hline & $(0.02385)$ & $(0.03488)$ & $(0.02499)$ & $(0.02458)$ & $(0.02959)$ & $(0.03032)$ \\
\hline & [0.73067] & [0.87930] & [0.45262] & {$[-0.13588]$} & {$[-1.29709]$} & {$[-1.60150]$} \\
\hline \multirow[t]{3}{*}{ Nikkei(-3) } & -0.033824 & -0.040971 & -0.053099 & -0.029131 & -0.009346 & -0.0064 \\
\hline & $(0.02384)$ & $(0.03486)$ & $(0.02498)$ & $(0.02457)$ & $(0.02957)$ & $(0.03031)$ \\
\hline & {$[-1.41863]$} & {$[-1.17522]$} & {$[-2.12560]$} & {$[-1.18578]$} & {$[-0.31602]$} & {$[-0.21115]$} \\
\hline \multirow[t]{3}{*}{ Nikkei(-4) } & -0.034497 & -0.014 & -0.027891 & -0.032889 & -0.05024 & -0.011803 \\
\hline & $(0.02375)$ & $(0.03472)$ & $(0.02488)$ & $(0.02447)$ & $(0.02946)$ & $(0.03019)$ \\
\hline & {$[-1.45262]$} & {$[-0.40317]$} & {$[-1.12092]$} & {$[-1.34408]$} & {$[-1.70552]$} & {$[-0.39098]$} \\
\hline \multirow[t]{3}{*}{ Nikkei(-5) } & 0.012416 & 0.020881 & -0.002206 & 0.023789 & -0.025856 & -0.005641 \\
\hline & $(0.02155)$ & $(0.03151)$ & $(0.02258)$ & $(0.02220)$ & $(0.02673)$ & $(0.02739)$ \\
\hline & [0.57619] & [0.66274] & {$[-0.09771]$} & [1.07142] & {$[-0.96738]$} & {$[-0.20593]$} \\
\hline \multirow[t]{3}{*}{$\operatorname{SSE}(-1)$} & -0.019515 & 0.010246 & 0.008770 & -0.030292 & -0.049499 & -0.035139 \\
\hline & $(0.02307)$ & $(0.03373)$ & $(0.02417)$ & $(0.02377)$ & $(0.02862)$ & $(0.02933)$ \\
\hline & {$[-0.84590]$} & [0.30374] & [0.36284] & {$[-1.27431]$} & [-1.72977] & {$[-1.19816]$} \\
\hline \multirow[t]{3}{*}{$\operatorname{SSE}(-2)$} & 0.031393 & 0.012331 & -0.017801 & 0.017471 & 0.039269 & 0.044956 \\
\hline & $(0.02313)$ & $(0.03382)$ & $(0.02423)$ & $(0.02383)$ & $(0.02869)$ & $(0.02940)$ \\
\hline & [1.35723] & [0.36460] & {$[-0.73453]$} & [0.73305] & [1.36872] & [1.52895] \\
\hline SSE $(-3)$ & 0.011506 & 0.025435 & -0.007313 & 0.026895 & 0.023558 & 0.029910 \\
\hline
\end{tabular}




\begin{tabular}{|c|c|c|c|c|c|c|}
\hline & DJIA & IBOVESPA & IPC & FTSE & Nikkei & SSE \\
\hline & $(0.02320)$ & $(0.03393)$ & $(0.02431)$ & $(0.02391)$ & $(0.02878)$ & (0.02950) \\
\hline & [0.49588] & [0.74965] & {$[-0.30080]$} & [1.12489] & [0.81852] & [1.01399] \\
\hline \multirow[t]{3}{*}{$\operatorname{SSE}(-4)$} & -0.01319 & -0.008662 & -0.037223 & -0.047156 & -0.062963 & -0.02813 \\
\hline & $(0.02315)$ & $(0.03385)$ & $(0.02425)$ & $(0.02385)$ & $(0.02872)$ & $(0.02943)$ \\
\hline & {$[-0.56976]$} & {$[-0.25589]$} & {$[-1.53464]$} & {$[-1.97690]$} & [-2.19268] & {$[-0.95587]$} \\
\hline \multirow[t]{3}{*}{ SSE $(-5)$} & -0.070413 & -0.10437 & -0.055398 & -0.051546 & -0.091459 & -0.026851 \\
\hline & $(0.02308)$ & $(0.03375)$ & $(0.02419)$ & $(0.02379)$ & $(0.02863)$ & (0.02935) \\
\hline & [-3.05023] & {$[-3.09208]$} & [-2.29043] & {$[-2.16706]$} & [-3.19406] & {$[-0.91500]$} \\
\hline \multirow[t]{3}{*}{ C } & 0.034606 & 0.003713 & 0.043612 & 0.019459 & 0.037564 & 0.041216 \\
\hline & $(0.02971)$ & $(0.04344)$ & $(0.03113)$ & $(0.03061)$ & $(0.03685)$ & $(0.03777)$ \\
\hline & [1.16483] & [0.08548] & [1.40110] & [0.63569] & [1.01937] & [1.09133] \\
\hline$R^{2}$ & 0.059264 & 0.038917 & 0.032652 & 0.055875 & 0.223750 & 0.056982 \\
\hline Adj. $R^{2}$ & 0.036919 & 0.016088 & 0.009675 & 0.033449 & 0.205312 & 0.034582 \\
\hline Sum sq. resids & 1417.963 & 3031.606 & 1556.550 & 1505.436 & 2181.634 & 2291.402 \\
\hline S.E. equation & 1.059573 & 1.549297 & 1.110145 & 1.091766 & 1.314284 & 1.346942 \\
\hline F-statistic & 2.652208 & 1.704729 & 1.421046 & 2.491562 & 12.13514 & 2.543878 \\
\hline $\begin{array}{l}\text { Log } \\
\text { likelihood }\end{array}$ & -1895.296 & -2386.933 & -1955.629 & -1934.026 & -2174.058 & -2205.819 \\
\hline Akaike AIC & 2.977273 & 3.737145 & 3.070524 & 3.037135 & 3.408126 & 3.457216 \\
\hline Schwarz sc & 3.101022 & 3.860893 & 3.194272 & 3.160883 & 3.531875 & 3.580964 \\
\hline $\begin{array}{l}\text { Mean } \\
\text { dependent }\end{array}$ & 0.035090 & -0.000978 & 0.037870 & 0.021946 & 0.050049 & 0.043279 \\
\hline $\begin{array}{l}\text { S.D. } \\
\text { dependent }\end{array}$ & 1.079691 & 1.561912 & 1.115555 & 1.110496 & 1.474317 & 1.370854 \\
\hline $\begin{array}{l}\text { Determinant re } \\
\text { (dof adj.) }\end{array}$ & esid covariance & 1.253348 & & & & \\
\hline Determinant re & esid covariance & 1.083643 & & & & \\
\hline Log likelihood & & -11068.61 & & & & \\
\hline Akaike informa & ation criterion & 17.39507 & & & & \\
\hline Schwarz criteri & & 18.13756 & & & & \\
\hline
\end{tabular}

Nota: Debajo de cada parámetro estimado se muestran los errores estándar y entre paréntesis el estadístico $t$ de Student. 
176 ECONOMÍA: TEORÍA Y PRÁCTICA • Nueva Época, número 43, julio-diciembre 2015

\section{Gráfica A1. Funciones impulso-respuesta (continúa)}
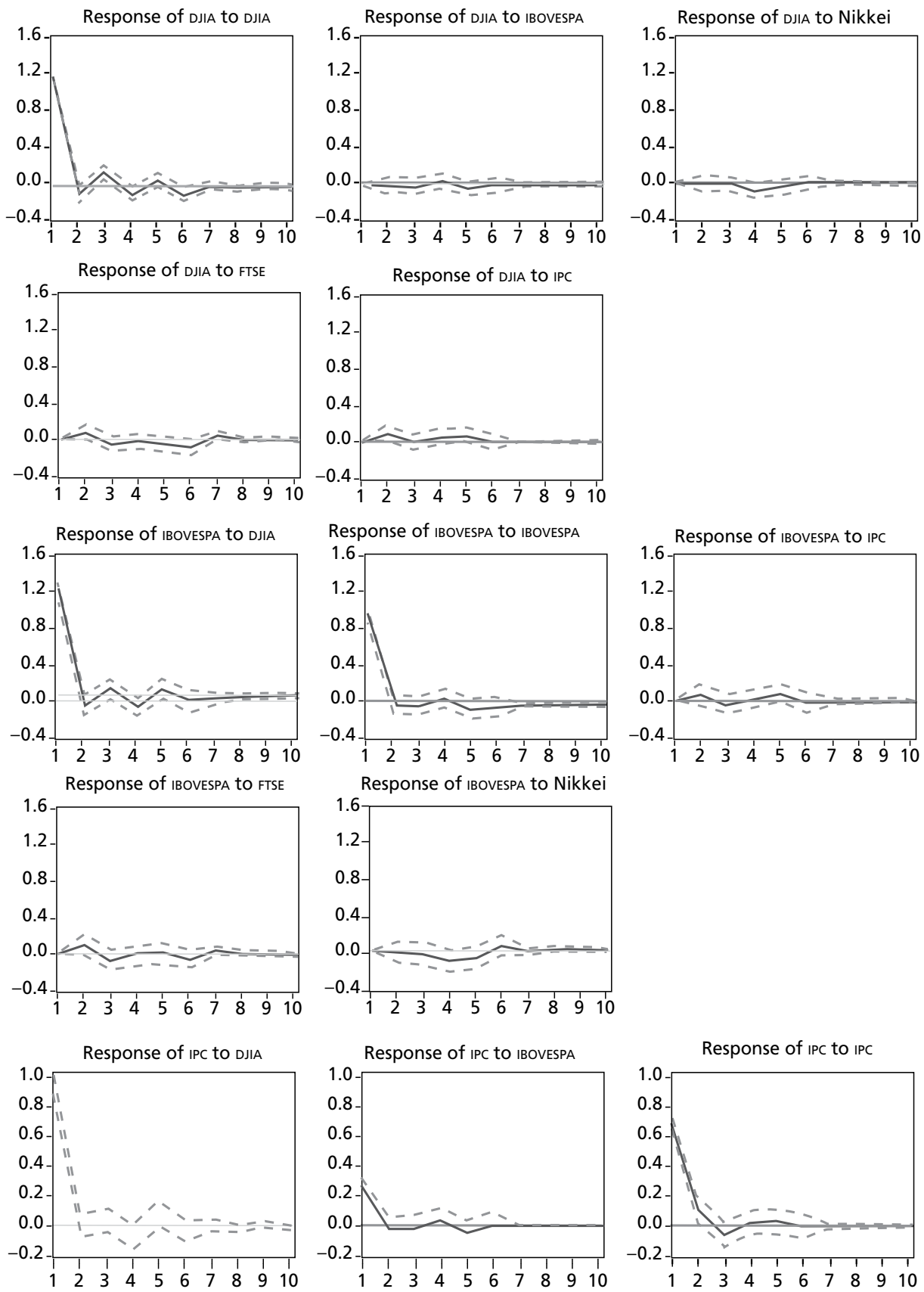


\section{Gráfica A1. Funciones impulso-respuesta (concluye)}
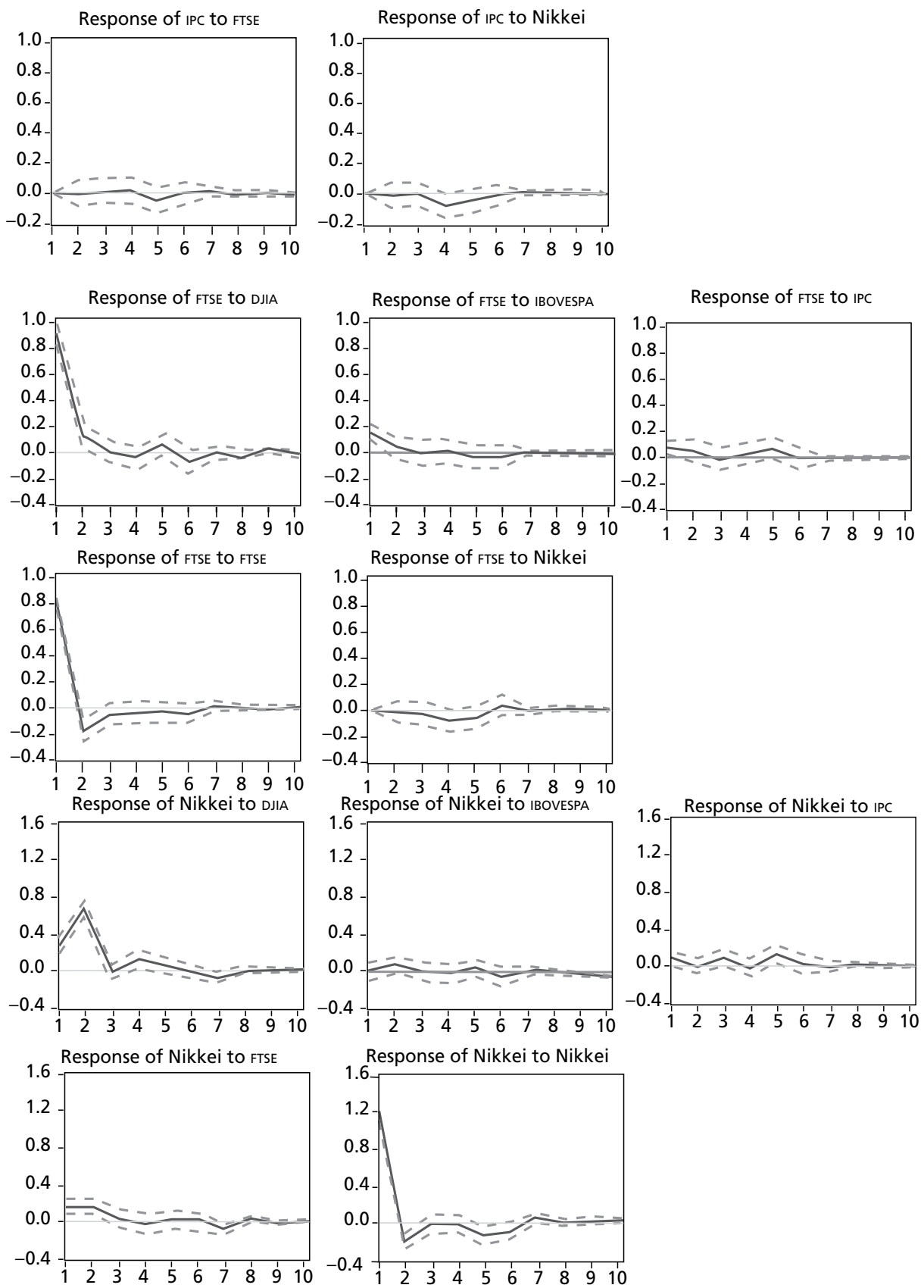


\section{REFERENCIAS BIBLIOGRÁFICAS}

Angkinand, Apanard Penny; Barth, James R., y Kim, Hyeongwoo (2009), "Spillover effects from the Us financial crisis: Some time-series evidence from national stock returns", en B. E. Gup (ed.), The financial and economic crises: an international perspective, s.l., Edward Elgar, pp. 24-52-

Arestis, Philip; Demetriadis, Panicos O., y Luintel, Kul B. (2001), "Financial development and economic growth: the role of stock markets", Journal of Money, Credit, and Banking, 33 (1), pp. 16-41.

Assaf, Ata (2003), "Transmission of stock price movements: The case of GCC stock markets", Review of Middle East Economics and Finance, 1 (2), pp 73-92.

Atje, Raymond, y Jovanovic, Boyan (1993), "Stock markets and development", European Economic Review, 37 (2-3), pp. 632-640.

Barajas, Adolfo; Beck, Thorsten; Dabla-Norris, Era, y Yousefi, Seyed Reza (2013), “Too cold, too hot, or just right? Assessing financial sector development across the globe", IMF Working Papers 13/130 (mayo).

Barajas, Adolfo; Chami, Ralph, y Yousefi, Seyed Reza (2013), "The finance and growth nexus re-examined: Do all countries benefit equally", IMF Working Papers $13 / 130$.

Bayraktar, Nihal (2014), "Measuring relative development level of stock markets: Capacity and effort of countries", Borsa Istanbul Review 14 (2), pp. 74-95.

Beck, Thorsten; Demirguc-Kunt, Asli, y Levine, Ross (2000), "A new database on the structure and development of the financial sector", The World Bank Economic Review, 14 (3), pp. 597-605.

- (2010) "Financial institutions and markets across countries and over time: the updated financial development and structure database", World Bank Economic Review, 24 (1), 77-92.

Beck, Thorsten, y Demirgüç-Kunt, Asli (2009), "Financial institutions and markets across countries and over time", World Bank Policy Research Paper 4943.

Bessler, David, y Yang, Jian (2003), "The structure of interdependence in international stock markets", Journal of Money and Finance, 22 (2), 261-287.

Billmeier, Andreas, y Massa, Isabella (2009), "What drives stock market development in emerging markets-institutions, remittances or natural resources?", Emerging Markets Review, 10 (1), pp. 23-35.

Brugger, Samuel, y Ortiz, Edgar (2012) "Mercados accionarios y su relación con la economía real en América Latina”, Problemas del Desarrollo, 43 (168) 63-93.

Chen, Gong-meng; Firth, Michael, y Rui, Oliver (2002), "Stock market linkages: eviden- 
ce from Latin America", Journal of Banking and Finance, 26 (6), pp. 11131141.

Cihak, Martin; Demirguc-Kunt, Asli; Feyen, Erick, y Levine, Ross (2012), "Financial development in 205 economies, 1960 to 2010", ponencia en la Conference on Financial Deepening, Macro-stability, and Growth, realizada por el Banco Mundial y el Fondo Monetario Internacional, Washington, DC, 24 de septiembre.

Climent, Francisco; Meneu, Vicente, y Pardo, Ángel (1999), "Influencia y sensibilidad de los mercados bursátiles europeos", Información Comercial Española, 0 (782), 77-87.

_ (2001), "Flujos de Información entre las principales áreas bursátiles", Universidad de Valencia. Consultado el 28 de marzo de 2014, en: http://www.uv. es/ fcliment/areas.pdf.

Demirguc-Kunt, Asli, y Levine, Ross (1996), "Stock markets, corporate finance and economic growth: an overview", The World Bank Economic Review, 10 (2), pp. 223-239.

Eun, Cheol S., y Shim, Sangdal (1989), "International Transmission of Stock Market Movements", The Journal of Financial and Quantitative Analysis, 24 (2), pp. 241-256.

Federación Internacional de Bolsas de Valores (2010), "International Stock Exchanges". Consultado el 14 de julio de 2014, en: http://www.world-exchanges.org/statistics/statistics-definitions.

Friedman, Joseph, y Shachmurove, Yochanan (1997), "Co-movements of major European community stock markets: a vector autoregression analysis", Global Finance Journal, 8 (2), pp. 257-277.

Forbes, Kristin J., y Chinn, Menzie D. (2004), "A Decomposition of Global Linkages in Finacial Markets Over Time", The Review of Economics and Statistics, 86 (3), pp. 705-722.

Garcia, Valeriano F; y Liu, Lin (1999), "Macroeconomic determinants of stock market development", Journal of Applied Economics, 2 (1), pp. $29-59$.

Gredenhoff, Mikael, y Karlson, Sune (1999), "Lag-length selection in VAR-models using equal and unequal lag-length procedures", Computational Statistics, 14 (2), 171-187.

Koch, Paul y Koch, Timothy (1991), "Evolution in dynamic linkages across daily national stock indexes", Journal of International Money and Finance, 0 (10), pp. 231-251.

Lanteri, Luis (2011), "Desarrollo del mercado accionario y crecimiento económico. Alguna evidencia para la Argentina", Ensayos de Economía, 0 (38), pp. 117-145. 
Levine, Ross, y Zervos, Sara (1998) "Stock markets, banks, and economic growth", American Economic Review, 88 (3), pp. 537-558.

Liu, Angela; Pan, Ming-Shiun, y Shieh, Joseph (1998), "International Transmission of Stock Price Movements: Evidence from the us and five Asian-Pacifc Markets", Journal of Economics and Finance, 22 (1), pp. 59-69.

Malkiel, Burton (2003), "Efficient Markets Hypothesis and its critics", Journal of Economic Perspectives, 17 (1), pp. 59-82.

Mills, Terence, y Mills, Alessandra (1991), “The International transmission of bond market movements", Bulletin of Economic Research, 43 (3), pp. 273-281.

Naceur, Samy Ben; Ghazouani, Samir, y Omran, Mohamed (2007), "The determinants of stock market development in the Middle-Eastern and North African Region", Managerial Finance, 33 (7), pp. 477-489.

Narayan, Paresh Kumar; Mishra, Sagarika, y Narayan, Seema (2011), "Do market capitalization and stocks traded converge? New global evidence", Journal of Banking \& Finance, 35 (10), pp. 2771-2781.

Peña, Ignacio (1991), Sobre la relación entre los mercados bursátiles internacionales y la bolsa de Madrid, Madrid, Universidad Carlos III.

Rigobon, Ehrmann, y Fratzcher, Marcel (2005) "Stock bonds, money markets and exchange rates: Measuring international financial transmission", European Central Bank Working Paper Series 452.

Runkle, David (1987), "Vector autoregressions and reality", Journal of Business and Economics Statistics, 5 (4), pp. 437-442.

Sims, Christopher (1980), "Macroeconomics and Reality", Econometrica, 48 (1), pp. $1-48$.

Soydemir, Gokce (2000), "International Transmission Mechanism of Stock Market Movements", Journal of Forecasting, 19 (3), pp. 149-176.

Tsangyao, Chang, y Chien-Chung, Nieh (2001), "International Transmission of Stock Price Movements among Taiwan and its Trading Partners", Pacific Basin Financial Markets and Policies, 4 (4), pp. 379-401.

Villanueva, Victoria (2008), “Contagio e interdependencias entre mercados de valores. Mecanismos y evidencias”, Comisión Nacional del Mercado de Valores. Consultado el 28 de marzo de 2014, en: www.cnmv.es

Walker, Eduardo (1998) "Mercado accionario y crecimiento económico en Chile", Cuadernos de Economía, 0 (104), pp. 49-72.

Wang, Jing; Medianu, Dana, y Whally, John (2011), "The contribution of China, India and Brazil to narrowing north-south differences in GDP/capita, world trade shares, and market capitalization”, NBER Working Paper Series 17681 (diciembre). 
Yang, Jian; Hsiao, Cheng; Li, Qi, y Wang, Zijun (2005), “The Emerging Market Crisis and Stock Market Linkages: Further Evidence", IEPR Working Papers 05.27.

Zavaleta, Osmar, y Urbina, Héctor (2011), "Mercados financieros y desarrollo económico: un análisis sobre México”, Ide@s Concyteg, 6 (68), pp. 226-235. 